\title{
DIABETES
}

\section{Breast cancer increases diabetes risk}

Postmenopausal women with breast cancer are more likely to develop diabetes mellitus than women without breast cancer, indicate new findings.

An association between diabetes mellitus and cancer has been previously shown. "This association has been partly attributed to shared risk factors, including obesity and insulin resistance," comments Lorraine Lipscombe, lead author of the study. Diabetes mellitus is known to increase breast cancer risk and breastcancer-related mortality; Lipscombe et al. set out to investigate whether patients with breast cancer are, in turn, at an increased risk of developing diabetes mellitus.

The researchers used population-based medical records from Ontario, Canada, to analyse the incidence of diabetes mellitus in postmenopausal women with $(n=24,976)$ and without $(n=124,880)$ a previous diagnosis of breast cancer. They also assessed diabetes mellitus incidence in the subgroup of women who received adjuvant chemotherapy $(n=4,404)$. The study spanned 12 years, with a mean follow-up period of 5.8 years.

The risk of diabetes mellitus was increased in women with breast cancer compared with controls, but only after 2 years of breast cancer diagnosis (HR 1.07). The risk continued to increase up to 10 years postdiagnosis (HR 1.21). However, among women who received chemotherapy the increase in diabetes mellitus risk was higher in the first 2 years after diagnosis (HR 1.24), decreasing afterwards. "This might be related to chemotherapy or, possibly, to glucocorticoid therapy, which is often used in this setting," says Lipscombe.

Increased screening and prevention of diabetes mellitus in patients with cancer may be warranted, conclude Lipscombe and colleagues.

Joana Osório

Original article Lipscombe, L. L. et al. Incidence of diabetes among postmenopausal breast cancer survivors. Diabetologia doi:10.1007/s00125-012-2793-9 